\title{
Symptomatic improvement with gluten restriction in irritable bowel syndrome: a prospective, randomized, double blinded placebo controlled trial
}

\author{
Vinay G Zanwar, Sunil V Pawar, Pravir A Gambhire, Samit S Jain, Ravindra G Surude, Vinaya B Shah, \\ Qais Q Contractor, Pravin M Rathi \\ Department of Gastroenterology, Topiwala National Medical College \& Bai Yamunabai Laxman Nair Ch Hospital, Mumbai, India
}

\begin{abstract}
Background/Aims: The existence of non-celiac gluten sensitivity has been debated. Indeed, the intestinal and extra-intestinal symptoms of many patients with irritable bowel syndrome (IBS) but without celiac disease or wheat allergy have been shown to improve on a gluten-free diet. Therefore, this study set out to evaluate the effects of gluten on IBS symptoms. Methods: We performed a double-blind randomized placebo-controlled rechallenge trial in a tertiary care hospital with IBS patients who fulfilled the Rome III criteria. Patients with celiac disease and wheat allergy were appropriately excluded. The participants were administered a gluten-free diet for 4 weeks and were asked to complete a symptom-based questionnaire to assess their overall symptoms, abdominal pain, bloating, wind, and tiredness on the visual analog scale $(0-100)$ at the baseline and every week thereafter. The participants who showed improvement were randomly assigned to one of two groups to receive either a placebo (gluten-free breads) or gluten (whole cereal breads) as a rechallenge for the next 4 weeks. Results: In line with the protocol analysis, 60 patients completed the study. The overall symptom score on the visual analog scale was significantly different between the two groups $(P<0.05)$. Moreover, the patients in the gluten intervention group scored significantly higher in terms of abdominal pain, bloating, and tiredness $(P<0.05)$, and their symptoms worsened within 1 week of the rechallenge. Conclusions: A gluten diet may worsen the symptoms of IBS patients. Therefore, some form of gluten sensitivity other than celiac disease exists in some of them, and patients with IBS may benefit from gluten restrictions. (Intest Res 2016;14:343-350)
\end{abstract}

Key Words: Controlled clinical trial; Irritable bowel syndrome; Glutens; Non-celiac gluten sensitivity; Diet, gluten-free

\section{INTRODUCTION}

Gluten is a protein mixture composed of glutelin and gliadin (a prolamin protein) in an approximate 1 to 1 ratio, occurring in the endosperm of wheat and other cereals. Gliadins, a group of proteins rich in proline and glutamine, have been identified as the main culprit in gluten intolerance.

\footnotetext{
Received April 18, 2016. Revised June 15, 2016.

Accepted June 16, 2016.

Correspondence to Vinay G Zanwar, Department of Gastroenterology, Topiwala National Medical College \&t Bai Yamunabai Laxman Nair Ch Hospital OPD Building 7th Floor, Mumbai Central, Mumbai 400008, India. Tel: +912223016139, Fax:+91-2223021168, E-mail: drvgzanwar@gmail.com

Financial support: None. Conflict of interest: None.
}

Apart from celiac disease (CD), wheat allergy and non-celiac gluten sensitivity (NCGS) represent the spectrum of glutenrelated disorders. $\mathrm{CD}$ is an immune mediated malabsorption syndrome, typically diagnosed in patients with diarrhea by the presence of villous atrophy in duodenal histology; positive serology for CD; and a rapid clinical or mucosal response to a gluten-free diet (GFD). ${ }^{1}$ However, NCGS is still a diagnosis of exclusion. ${ }^{2}$ Although the NCGS pathophysiology is currently far from clear, the available data suggest immune activation as a common denominator in both CD and NCGS. Patients often report intestinal and extra intestinal symptoms that may be indistinguishable from those reported by patients with IBS. ${ }^{3}$ The International Expert Meeting on Gluten Related Disorders defined NCGS as the occurrence

\footnotetext{
๑ Copyright 2016. Korean Association for the Study of Intestinal Diseases. All rights reserved.

This is an Open Access article distributed under the terms of the Creative Commons Attribution Non-Commercial License (http://creativecommons.org/licenses/by-nc/4.0)

which permits unrestricted non-commercial use, distribution, and reproduction in any medium, provided the original work is properly cited.
} 
of intestinal and extra intestinal symptoms such as tiredness, headache, anxiety, and numbness after ingestion of gluten containing food in subjects without wheat allergy or $\mathrm{CD}{ }^{4}$

The prevalence of CD in diarrhea-predominant IBS is similar to that of healthy controls at approximately $0.4 \%{ }^{5}$ The overall prevalence of the condition is, however, difficult to estimate owing to heightened public awareness and selfrestriction of gluten in diet. ${ }^{6,7}$ In the United State, the prevalence of self-prescribed GFD in an unselected population of subjects aged 6 years or older was reported as $0.5 \% .^{8}$ In a double-blind placebo-controlled challenge (DBPC) of patients affected by IBS, the prevalence of gluten sensitivity was reported to be up to $28 \%{ }^{9}$ A recent study has also reported an improvement in IBS symptoms after 6 weeks of GFD. ${ }^{10}$

Dietary restriction as a therapeutic strategy in the management of IBS has been investigated rigorously in the west. However, perceived as a "Western disease," gluten sensitivity has not been taken seriously in India. GFD means more than giving up bread and chapattis, as many Indian foods contain hidden gluten in their ingredients. Hence, it is difficult to estimate exactly the gluten-sensitive population other than CD.

The aim of this study was to evaluate the effect of gluten on gastrointestinal symptoms in Indian IBS patients.

\section{METHODS}

\section{Plan of Research}

The study was conducted from December 2013 to July 2015 at a tertiary health care center in the gastroenterology outpatient clinic affiliated to a University Medical College in Mumbai, India. The effects of a daily dose of gluten versus placebo in IBS patients were compared in a prospective, randomized, DBPC rechallenge trial. All participants gave written informed consent before the initiation of study procedures.

\section{Characteristics of Subjects}

Inclusion and exclusion criteria: patients aged $>16$ years, with symptoms of IBS as per the Rome III criteria, and willing to adhere to the prescribed diet, were included. Patients underwent serologic study, esophagogastroduodenoscopy, and colonoscopy at enrolment. An IgA anti-tissue transglutaminase (anti-tTg) assay was performed using an enzyme immunoassay (AESKULISA kit; AESKU.Diagnostics, Wendelsheim, Germany), with a biological reference value of $>18.0 \mathrm{U} / \mathrm{mL}$. In accordance with a standard diagnostic protocol, four to six biopsy specimens were taken from the second part of duodenum using an end-viewing endoscope (TGF150; Olympus, Tokyo, Japan) by a single endoscopist. Biopsies were embedded in paraffin, sections prepared, stained with $\mathrm{H} \& \mathrm{E}$, and examined by a single pathologist for modified Marsh grading, with an upper limit of 40 intraepithelial lymphocyte per 100 enterocytes. An HLA-DQ haplotyping was not performed. Patients with CD (positive serological test for IgA anti-tTg antibody and duodenal villous atrophy while on a gluten-containing diet) and wheat allergy (positive IgE-mediated immune allergy test to wheat) were excluded. Additional exclusion criteria were: patients already on GFD; presence of cirrhosis or inflammatory bowel disease; excessive alcohol intake; patients currently prescribed and using systemic immunosuppressants, nonsteroidal anti-inflammatory agents, or medications affecting gastrointestinal motility; abnormal thyroid function tests; presence of a psychiatric disease; pregnancy; and inability to give written informed consent.

\section{Study Protocol}

Patients were asked to complete a baseline and weekly follow-up questionnaires assessing the severity of abdominal pain, bloating, wind, tiredness, and overall symptoms. These symptoms were graded on a 100-mm visual analogue scale (VAS), with 0 and 100 representing no or severe symptoms, respectively. Patients usually consumed flat breads in their daily meals, amounting to 25 to $30 \mathrm{~g}$ of gluten/day. At the beginning of the study, a dietician provided advice to all patients on following a GFD for 4 weeks. They were asked to restrain from wheat, rye, bakery products, pastas, noodles, graham flour, drinks like beer, and potential sources of hidden gluten such as candy, gravy, sauce, and lipstick. Instead, they were encouraged to include millet, milo (jowar), soybean, maize, rice, and fruits in their diet. They were also asked to note dietary habits and maintain food diaries. Symptoms were recorded on VAS during weekly follow-ups. Patients who responded adequately to the GFD and had improvement in their symptoms, defined as a $30 \%$ decrease in symptom VAS from the baseline for at least $50 \%$ of the time, were included in the study. Patients who did not respond to the GFD were allowed to withdraw from the study. After 4 weeks of the washout (elimination diet) period, the responding patients were randomly assigned into two groups for a DBPC rechallenge. The patients in the gluten group consumed two slices of bread containing gluten, while the patients in the placebo group consumed two slices of gluten- 
free breads, each morning for the course of the rechallenge. The rechallenge breads were provided to the patients during their weekly follow-ups, and were similar in texture and appearance. The DBPC rechallenge lasted 4 weeks and the patients were advised to maintain GFD throughout this period. A dietician advised the patients on macronutrient and caloric intake based on their sex and physical activity. At each weekly follow-up, dietary adherence was evaluated through questioning and food diaries. The study was approved by Institutional Ethics Committee.

\section{Outcome/End Points}

The primary outcome was a change in overall symptom VAS. Secondary outcomes were changes in individual abdominal symptoms VAS, as assessed at the weekly followups.

\section{Sample Size}

Sample size was calculated using the formula for two parallel-sample means:

$$
\mathrm{n}_{1}=\mathrm{n}_{2}=\left[\left(\mathrm{Z}_{\alpha / 2}+\mathrm{Z}_{\beta}\right)^{2} \times \sigma^{2}\right] /\left(\mu_{2}-\mu_{1}\right)^{2},
$$

with $\alpha=0.05, \beta=0.20$, where $\mu_{2}-\mu_{1}$ is the acceptable mean difference between a test drug $\left(\mu_{2}\right)$ and a placebo control or active control agent $\left(\mu_{1}\right)$. With the overall symptom VAS reference values of $7.4 \pm 1.31$ at baseline, and $6.25 \pm 1.52$ after GFD, ${ }^{11}$ the calculated sample size was 48 (24 in each group). With an estimated drop-out rate of $10 \%$, the final sample size required was 54 ( 27 in each group).

\section{Randomization}

Patients were randomized by an independent observer according to a computer-generated list of random numbers. Both patients and investigators were blinded to the study treatment.

\section{Statistical Methods}

Qualitative data were represented as frequencies and percentages. Among the qualitative data, nominal data included the group categories; sex; esophagogastroduodenoscopy findings; and duodenal biopsy findings, and the ordinal data included VAS scores for abdominal pain; bloating; wind; tiredness; and overall symptoms. Data were assessed for normality of distribution. Variables were measured during the gluten washout period and after the rechallenge trial. As- sociation between qualitative variables was assessed using a chi-square test with a Yate's continuity correction for $2 \times 2$ contingency tables, or using a Fisher exact test where the chi-square test was not valid due to small counts. The nonnormally distributed ordinal data were compared between the two groups using a Mann-Whitney $U$ test (e.g., tiredness VAS in the gluten and placebo groups at fourth rechallenge week).

Quantitative data were represented as mean $\pm \mathrm{SD}$ or median and interquartile range, and included age; duration of illness; BMI; ESR; and IgA anti-tTG antibody levels. Data were tested for normality using the Shapiro-Wilk test, and the quantitative data were compared between the qualitative variables and the study subgroups using an unpaired $t$ test (for normally distributed data) or a Mann-Whitney $U$ test (for non-normally distributed data, e.g., age comparison between the gluten and placebo groups).

Comparison between ordinal data measured at five occasions during the study period (after 4 weeks of GFD, and after first, second, third, and fourth week of rechallenge) was done separately for the gluten group using the Friedman's repeated measures analysis of variance on ranks. Pairwise multiple comparisons were done using a Tukey test when the Friedman test was statistically significant. Appropriate statistical software, including, but not restricted to MS Excel and PSPP version 0.8.5 (Free Software Foundation Inc., Boston, MA, USA), were used for analysis. Results were graphically represented, where deemed necessary, using MS Excel 2010 (Microsoft, Redmond, WA, USA).

\section{RESULTS}

\section{Demographic Characteristics}

The study flow chart is shown in Fig. 1. Sixty-five patients underwent randomization in this DBPC rechallenge trial; 34 received gluten and 31 received placebo. Five patients could not complete the trial as they were unable to keep weekly follow-ups. Demographic data, clinical characteristics, and laboratory parameters of the gluten and placebo groups are summarized and compared in Table 1; both groups were comparable $(P>0.05)$.

\section{Change in Symptom Severity Post Rechallenge}

Out of the initial group of 180 patients, overall symptom VAS improved for 65 patients after GFD. After 4 weeks of food rechallenge, patients in the gluten group exhibited 


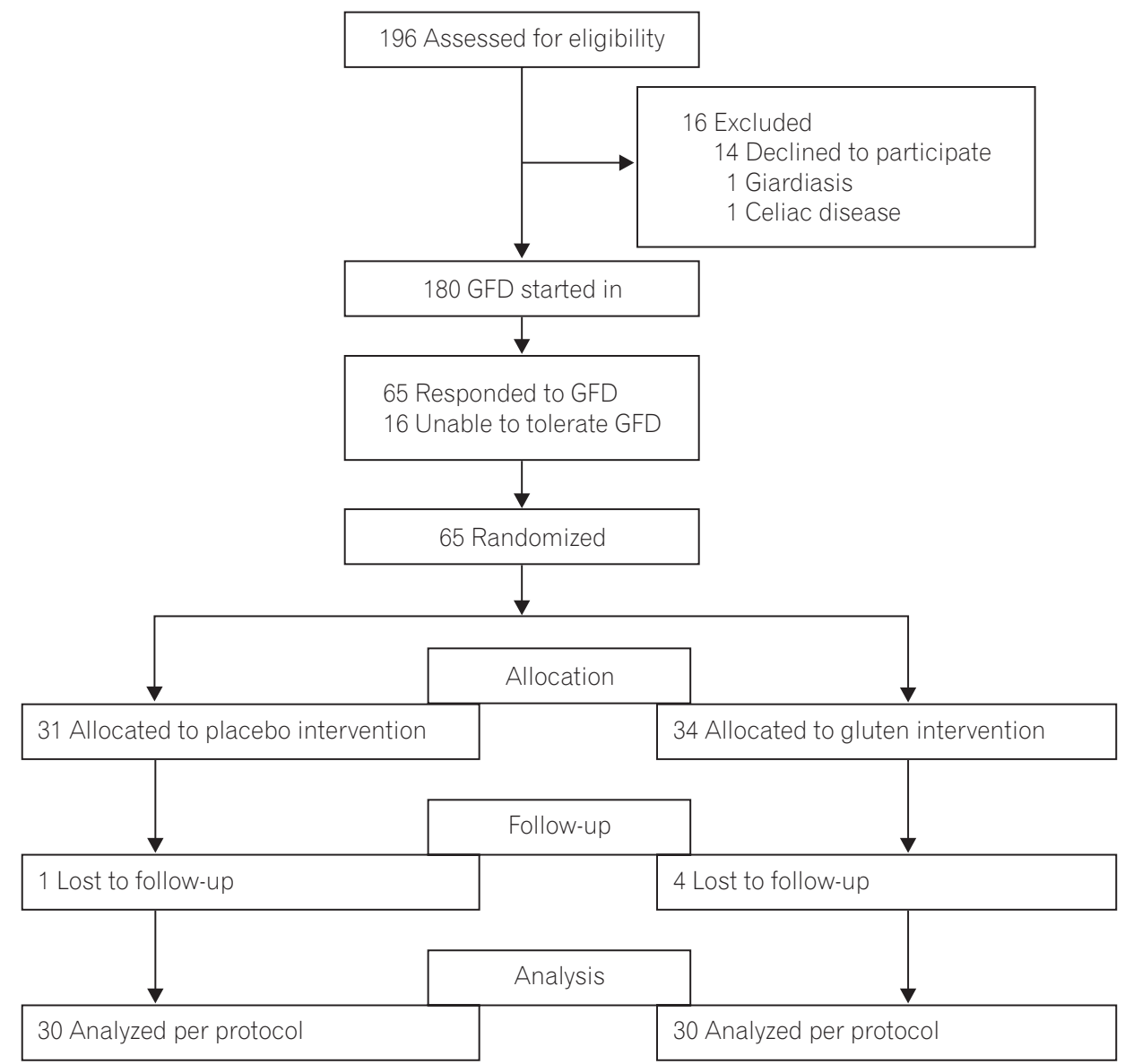

Fig. 1. Participants recruitment flow chart showing screening failure and withdrawals. GFD, gluten-free diet.

Table 1. Patient Characteristic according to Dietary Intervention Group

\begin{tabular}{lccc}
\hline \multicolumn{1}{c}{ Patient characteristic } & Gluten $(\mathbf{n = 3 0})$ & Placebo $(\mathbf{n = 3 0})$ & P-value \\
\hline Age $(\mathrm{yr})$ & $37(18-60)$ & $35(18-56)$ & 0.514 \\
Male sex & $17(56)$ & $18(60)$ & 0.793 \\
BMl $\left(\mathrm{kg} / \mathrm{m}^{2}\right)$ & $21.35(18.00-26.40)$ & $21.50(19.00-32.00)$ & 0.773 \\
Duration of illness $(\mathrm{yr})$ & $4.27 \pm 2.85$ & $4.67 \pm 2.92$ & 0.455 \\
IgA tTG antibody level $(\mathrm{U} / \mathrm{mL})$ & $3.83 \pm 1.71$ & $3.85 \pm 1.51$ & 0.728 \\
ESR & $13.60 \pm 3.12$ & $13.80 \pm 3.26$ & 0.831 \\
\hline
\end{tabular}

Values are presented as median (range), number (\%), or mean \pm SD.

tTG, tissue transglutaminase.

worsening of symptoms, with significantly higher weekly median overall symptom VAS, compared to those in the placebo group $(P<0.05)$ (Fig. 2).

Secondary outcome measures were to assess the change in individual gastrointestinal symptom VAS. During the entire rechallenge period, the median VAS scores for abdominal pain, bloating, and tiredness were significantly higher in the gluten group compared to the placebo (Fig. 3). Patients in the gluten group showed significantly higher changes in the abdominal pain, bloating, and tiredness VAS scores from the fourth week of GFD to each of the 4 rechallenge weeks, compared to the patients in the placebo group $(P<0.05)$. However, VAS score for wind was not significantly different in both groups following the rechallenge $(P>0.05)$.

Within the gluten group, the VAS scores for abdominal pain, bloating, wind, tiredness, and overall symptoms, differed significantly over the entire study period based on the Friedman's repeated measures analysis of variance on ranks. Moreover, these differences were maintained following post hoc analysis for multiple pairwise comparisons $(P<0.05)$. 


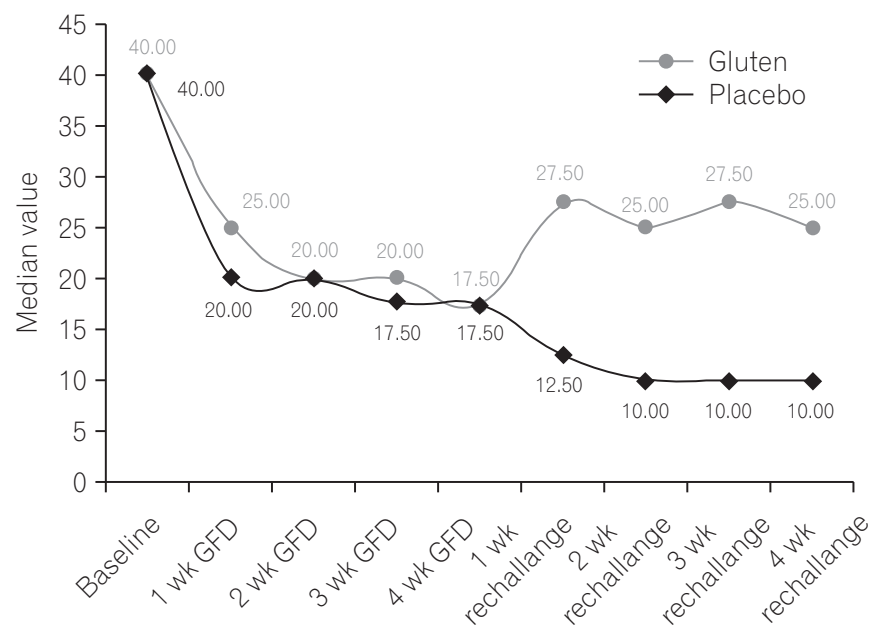

Overall VAS

Fig. 2. Overall symptom visual analogue scale (VAS) score between gluten and placebo groups at various time points during the study. GFD, gluten-free diet.
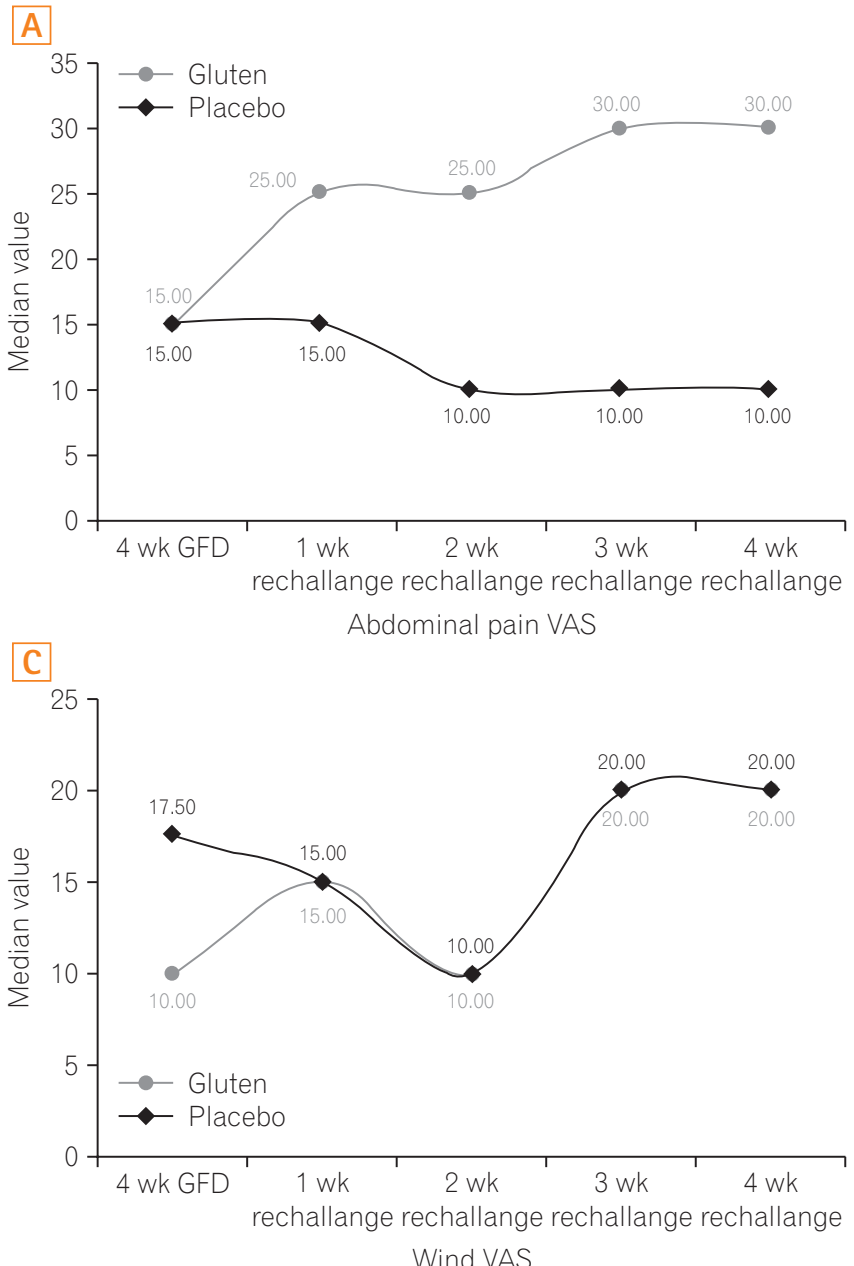

The placebo group also showed similar changes in the VAS symptom scores over the study period; however, only the changes in abdominal pain and wind VAS scores after rechallenge remained significantly different following post hoc analysis $(P<0.05)$.

\section{DISCUSSION}

The present DBPC gluten rechallenge study was performed in Indian IBS patients. We used two slices of glutencontaining bread for a controlled rechallenge trial. Although this study used a lower dose of gluten as compared to some other studies, ${ }^{9,12}$ it was consistent with recent trials for gluten challenge where this dose was regarded sufficient to induce symptoms in NCGS patients. ${ }^{12,13}$ Further, a 14 day dose of $\geq 3$ $\mathrm{g}$ of gluten/day has been shown to induce histological and serological changes in a majority of CD-affected adults. ${ }^{14}$
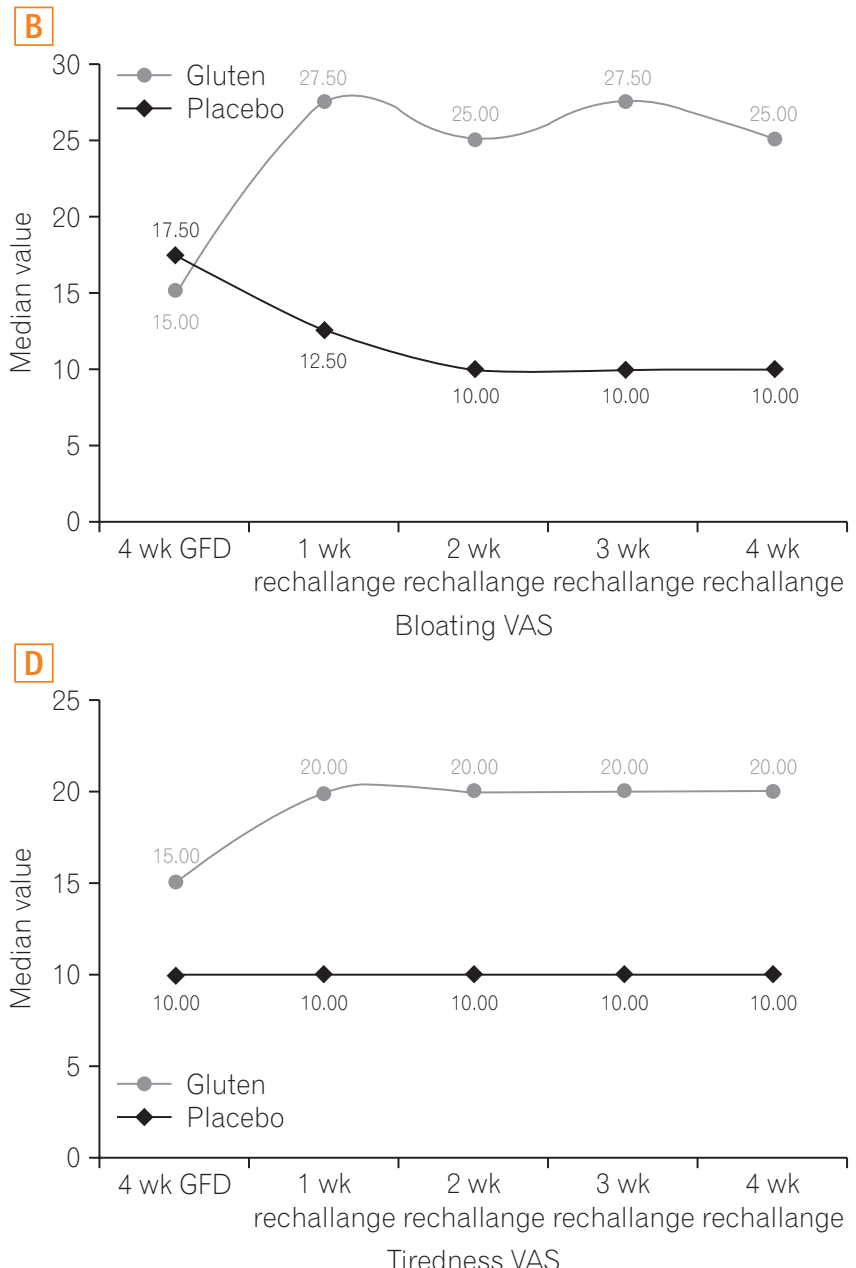

Fig. 3. Comparison of (A) abdominal pain, (B) bloating, (C) wind, and (D) tiredness visual analogue scale (VAS) scores in the rechallenge period in gluten and placebo groups. GFD, gluten-free diet. 
Overall symptom score in our study was significantly higher in the gluten group as compared to the placebo group. Worsening of overall symptoms was recorded in $55.67 \%$ of patients in the gluten group, compared to $33.3 \%$ of patients in the placebo group.

Among all participants in the trial, bloating and pain were the most reported symptoms, and these symptoms worsened most after gluten rechallenge (80\% and $73.33 \%$, respectively). Both bloating and pain have been reported as the highest scored symptoms in other previous studies. ${ }^{9,13,15}$ Further, the most significant differences in these gastrointestinal symptoms in the gluten group were observed between fourth week of GFD and the first week of rechallenge. Over the next rechallenge weeks, the scores were not statistically different, though marginally higher. This increase in symptoms within days of exposure to gluten strongly indicates a presence of gluten sensitivity. Similar observations have been reported in the past. ${ }^{9,15}$ In the placebo group, the differences in the individual symptom severities were not clinically apparent during each rechallenge week, and the symptom scores reached less severe values compared to those in the gluten group.

Among the systemic symptoms, tiredness showed the most significant difference between both the intervention groups. Increase in tiredness in the gluten group might suggest systemic effects of gluten in the sensitive patients. As tiredness is a common feature of IBS, ${ }^{16}$ its worsening by gluten may provide insights into a mechanism besides the altered intestinal mucosal permeability. Similar opinion regarding tiredness has been expressed by others in previous studies. ${ }^{9,15}$ A study by Biesiekierski et al. ${ }^{9}$ attempted to identify an immunological basis for the intestinal inflammation and permeability due to gluten. However, they noted that CRP, fecal lactoferrin levels, and dual sugar absorption test for permeability were similar between the gluten and placebo groups.

In patients with diarrhea-predominant IBS, significantly greater effect of gluten on the frequency of daily bowel movements and small bowel permeability (based on 0-2 hours levels of mannitol and lactulose to mannitol ratio) has been reported, compared to rice. ${ }^{17}$ Another study reported normalized stool frequency and gastrointestinal symptom scores in $60 \%$ of the patients following 6 months of GFD. ${ }^{18}$

The role of non-gliadin proteins in causing intestinal symptoms needs to be further explored. Of note, the amylase trypsin inhibitor enzyme family is known to activate the toll-like receptor-4 pathway of the innate immune system, stimulating the release of proinflammatory cytokines. ${ }^{19}$ This leads to poor absorption of carbohydrates, especially fructans, which are known to induce functional gut symptoms. ${ }^{20}$ A previous placebo-controlled cross-over rechallenge study found no evidence of specific or dose dependent effects of gluten on patients with NCGS placed on diets low in fermentable oligosaccharides, disaccharides, monosaccharide and polyols (FODMAPs). ${ }^{12}$ However according to the Salerno Experts' Criteria for NCGS, FODMAPs do not account for the extra intestinal manifestations. ${ }^{4}$

The prevalence of IBS in the Indian community is approximately $4 \%$, posing a significant burden on the rural adults. ${ }^{21}$ Its pathogenesis is not completely understood. Food plays a key role in IBS, and more than $60 \%$ of patients with IBS report the onset or worsening of symptoms after meals. ${ }^{22}$ Most IBS patients (84\%) report meal related symptoms to at least one food item, which are associated with higher symptom severity scores and a reduced quality of life. ${ }^{23-25}$ Gibson ${ }^{26}$ postulated three pathogenic mechanisms by which food items might induce abdominal symptoms in functional bowel disorders, via immune activation/mast cell pathway (food hypersensitivity), direct action of bioactive molecules (food chemicals), and luminal distension.

Symptoms of CD overlap with those of functional bowel diseases. A study in Indian patients with IBS reported a $0.8 \%$ prevalence of CD and a $5.2 \%$ prevalence of potential CD, highlighting the relationship between IBS and gluten sensitivity. ${ }^{27}$ However, the hypothesis that gluten is able to induce IBS-like symptoms in non-celiac patients is not new, ${ }^{28,29}$ and the possibility of a causal relationship between the ingestion of gluten and the occurrence of symptoms in the absence of CD or wheat allergy, referred to as NCGS, has attracted a lot of interest recently.

Although the prevalence of NCGS is not clearly defined yet, indirect evidence suggests that it is more common than $\mathrm{CD}^{4}$ At present, it is not clear whether NCGS is transient or permanent, how long the GFD should be continued, how to check the response to GFD, and whether there are any longterm consequences of GFD. Gluten tolerance may possibly need to be reassessed periodically in patients with NCGS. ${ }^{30}$ Both NCGS and non-celiac wheat sensitivity (NCWS) have created a complex scenario. Symptom induction by shortchain carbohydrates such as fructans and galacto-oligosaccharides (not gluten) in wheat is referred to as NCWS. It is possible that the terminology "NCGS" could be changed to "NCWS" in the near future. This would then exclude other relevant cereals like barley and rye. ${ }^{4}$ Recent data show a strong tendency of the NCWS patients toward autoimmunity, characterized by both associated autoimmune diseases 
and the presence of serum antinuclear antibodies, which, in turn, were correlated with the HLA DQ2/DQ8 haplotypes. ${ }^{31}$ There is an urgent need for validated biomarker to diagnose the condition with acceptable sensitivity and specificity. Our study is limited by the small sample size and short follow-up.

In conclusion, in patients with IBS, gluten triggers intestinal and systemic symptoms, and the symptom exacerbations occur mostly within a week of gluten rechallenge. Further confirmation of these findings in multi-centric studies will aid in understanding the benefit of gluten restriction in IBS patients.

\section{REFERENCES}

1. Green PH, Jabri B. Coeliac disease. Lancet 2003;362:383-391.

2. Elli L, Branchi F, Tomba C, et al. Diagnosis of gluten related disorders: celiac disease, wheat allergy and non-celiac gluten sensitivity. World J Gastroenterol 2015;21:7110-7119.

3. Drossman DA, Camilleri M, Mayer EA, Whitehead WE. AGA technical review on irritable bowel syndrome. Gastroenterology 2002;123:2108-2131.

4. Catassi C, Elli L, Bonaz B, et al. Diagnosis of non-celiac gluten sensitivity (NCGS): the Salerno Experts' Criteria. Nutrients 2015;7:4966-4977.

5. Cash BD, Rubenstein JH, Young PE, et al. The prevalence of celiac disease among patients with nonconstipated irritable bowel syndrome is similar to controls. Gastroenterology 2011;141:1187-1193.

6. Simpson S, Lebwohl B, Lewis SK, Tennyson CA, Sanders DS, Green PH. Awareness of gluten related disorders: a survey of the general public, chefs and patients. E Spen Eur E J Clin Nutr Metab 2011;6:e227-e231. doi:10.1016/j.eclnm.2011.08.001.

7. Rostami K, Hogg-Kollars S. A patient's journey: non-coeliac gluten sensitivity. BMJ 2012;345:e7982. doi:10.1136/bmj.e7982.

8. DiGiacomo DV, Tennyson CA, Green PH, Demmer RT. Prevalence of gluten-free diet adherence among individuals without celiac disease in the USA: results from the Continuous National Health and Nutrition Examination Survey 2009-2010. Scand J Gastroenterol 2013;48:921-925.

9. Biesiekierski JR, Newnham ED, Irving PM, et al. Gluten causes gastrointestinal symptoms in subjects without celiac disease: a double-blind randomized placebo-controlled trial. Am J Gastroenterol 2011;106:508-514.

10. Volta U, Tovoli F, Cicola R, et al. Serological tests in gluten sensitivity (nonceliac gluten intolerance). J Clin Gastroenterol 2012;46:680-685.
11. Massari S, Liso M, De Santis L, et al. Occurrence of nonceliac gluten sensitivity in patients with allergic disease. Int Arch Allergy Immunol 2011;155:389-394.

12. Biesiekierski JR, Peters SL, Newnham ED, Rosella O, Muir JG, Gibson PR. No effects of gluten in patients with self-reported non-celiac gluten sensitivity after dietary reduction of fermentable, poorly absorbed, short-chain carbohydrates. Gastroenterology 2013;145:320-328.e3.

13. Di Sabatino A, Volta U, Salvatore C, et al. Small amounts of gluten in subjects with suspected nonceliac gluten sensitivity: a randomized, double-blind, placebo-controlled, cross-over trial. Clin Gastroenterol Hepatol 2015;13:1604-1612.e3.

14. Leffler D, Schuppan D, Pallav K, et al. Kinetics of the histological, serological and symptomatic responses to gluten challenge in adults with coeliac disease. Gut 2013;62:996-1004.

15. Shahbazkhani B, Sadeghi A, Malekzadeh R, et al. Non-celiac gluten sensitivity has narrowed the spectrum of irritable bowel syndrome: a double-blind randomized placebo-controlled trial. Nutrients 2015;7:4542-4554.

16. Piche T, Huet PM, Gelsi E, et al. Fatigue in irritable bowel syndrome: characterization and putative role of leptin. Eur J Gastroenterol Hepatol 2007;19:237-243.

17. Vazquez-Roque MI, Camilleri M, Smyrk T, et al. A controlled trial of gluten-free diet in patients with irritable bowel syndromediarrhea: effects on bowel frequency and intestinal function. Gastroenterology 2013;144:903-911.e3.

18. Wahnschaffe U, Schulzke JD, Zeitz M, Ullrich R. Predictors of clinical response to gluten-free diet in patients diagnosed with diarrhea-predominant irritable bowel syndrome. Clin Gastroenterol Hepatol 2007;5:844-850.

19. Junker Y, Zeissig S, Kim SJ, et al. Wheat amylase trypsin inhibitors drive intestinal inflammation via activation of toll-like receptor 4. J Exp Med 2012;209:2395-2408.

20. Shepherd SJ, Parker FC, Muir JG, Gibson PR. Dietary triggers of abdominal symptoms in patients with irritable bowel syndrome: randomized placebo-controlled evidence. Clin Gastroenterol Hepatol 2008;6:765-771.

21. Ghoshal UC, Abraham P, Bhatt C, et al. Epidemiological and clinical profile of irritable bowel syndrome in India: report of the Indian Society of Gastroenterology Task Force. Indian J Gastroenterol 2008;27:22-28.

22. Simrén M, Månsson A, Langkilde AM, et al. Food-related gastrointestinal symptoms in the irritable bowel syndrome. Digestion 2001;63:108-115.

23. Böhn L, Störsrud S, Törnblom H, Bengtsson U, Simrén M. Selfreported food-related gastrointestinal symptoms in IBS are common and associated with more severe symptoms and reduced quality of life. Am J Gastroenterol 2013;108:634-641. 
24. El-Salhy M, Ostgaard H, Gundersen D, Hatlebakk JG, Hausken $\mathrm{T}$. The role of diet in the pathogenesis and management of irritable bowel syndrome (review). Int J Mol Med 2012;29:723-731.

25. Zigich S, Heuberger R. The relationship of food intolerance and irritable bowel syndrome in adults. Gastroenterol Nurs 2013;36:275-282.

26. Gibson PR. Food intolerance in functional bowel disorders. J Gastroenterol Hepatol 2011;26 Suppl 3:128-131.

27. Sharma H, Verma AK, Das P, Dattagupta S, Ahuja V, Makharia GK. Prevalence of celiac disease in Indian patients with irritable bowel syndrome and uninvestigated dyspepsia. J Dig Dis 2015;16:443-448.
28. Ellis A, Linaker BD. Non-coeliac gluten sensitivity? Lancet 1978;1:1358-1359.

29. Cooper BT, Holmes GK, Ferguson R, Thompson RA, Allan RN, Cooke WT. Gluten-sensitive diarrhea without evidence of celiac disease. Gastroenterology 1980;79(5 Pt 1):801-806.

30. Fasano A, Sapone A, Zevallos V, Schuppan D. Nonceliac gluten sensitivity. Gastroenterology 2015;148:1195-1204.

31. Carroccio A, D’Alcamo A, Cavataio F, et al. High proportions of people with nonceliac wheat sensitivity have autoimmune disease or antinuclear antibodies. Gastroenterology 2015;149:596603.e1. 